\title{
INVESTIGACIONES
}

\section{Evolución de la segregación socioeconómica de las escuelas de América Latina}

\author{
The evolution of socio-economic segregation of Latin American schools
}

\author{
F. Javier Murillo ${ }^{a}$ Cynthia Duk ${ }^{b}$ Cynthia Martínez Garrido \\ ${ }^{a}$ Universidad Autónoma de Madrid. \\ Correo electrónico: javier.murillo@uam.es \\ ${ }^{b}$ Universidad Central de Chile. \\ Correo electrónico: cduk@ucentral.cl. \\ ${ }^{c}$ Universidad Autónoma de Madrid. \\ Correo electrónico: cynthia.martinez@uam.es.
}

\begin{abstract}
RESUMEN
El objetivo de esta investigación es determinar la evolución de la magnitud de la segregación escolar por nivel socioeconómico, tanto en su dimensión de uniformidad como de exposición, en algunos países de América Latina. Para ello se realiza una explotación de los datos de las seis ediciones PISA. La muestra está conformada por 374.490 estudiantes de 15.500 escuelas de 10 países latinoamericanos. Los resultados indican que, en seis de los ocho países con suficientes datos, la segregación parece tener una tendencia a la baja, especialmente en Argentina y Brasil. Esta disminución es muy discreta en comparación con la alta segregación que presentan. Además, en la mayoría de los países en que la segregación disminuye, lo hace en los estudiantes de familias de mayor nivel socioeconómico, incrementándose en los de menor. Es indispensable, por tanto, incorporar el debate sobre segregación escolar en la agenda de las políticas educativas públicas para tomar medidas que contribuyan a reducirla.
\end{abstract}

Palabras clave: segregación escolar, Inequidad, Sistemas educativos, Nivel socioeconómico, Resultados PISA.

\section{ABSTRACT}

The objective of this research was to determine the evolution of the magnitude of school segregation by socioeconomic level in some Latin American countries, both in terms of uniformity and exposure. For this purpose, data mining was carried out using the six versions of the PISA. The sample consisted of 374,490 students from 15,500 schools in 10 Latin American countries. The results indicate that, in six of the eight countries for which sufficient data existed, segregation seems to have a downward trend, especially in Argentina and Brazil. This decrease is subtle in comparison with the high overall rates of segregation that these countries present. Moreover, in the majority of the countries in which segregation decreases, it does so among students of higher socioeconomic level, increasing meanwhile for those at lower levels. It is essential, therefore, to incorporate the debate on school segregation in the agenda of public education policies to take measures that will contribute to reduce it.

Keywords: school segregation, inequity, educational systems, socioeconomic level, PISA results. 


\section{INTRODUCCIÓN}

América Latina es, en la actualidad, la región más inequitativa del mundo. ¿Qué están haciendo sus sistemas educativos para revertir esa situación?

No cabe duda de que la escolarización prácticamente universal alcanzada en pasadas décadas en niveles obligatorios ha supuesto un avance importante, pero está claro que no es suficiente. Si todos los niños, niñas y adolescentes no tienen una educación equitativa y de calidad, difícilmente se cumplirá el principio de igualdad de oportunidades educativas, esencial para conseguir una mayor justicia social. La segregación escolar, fenómeno por el cual los estudiantes se concentran de forma desigual en las escuelas en función de sus características o condiciones, es un factor que impide que todos los estudiantes reciban una educación equitativa y de calidad.

Tenemos suficientes evidencias para afirmar que los sistemas educativos de América Latina se encuentran entre los más segregados del mundo. Lo que agrava el hecho de que la educación que ofrecen las escuelas localizadas en los sectores más desfavorecidos es, por lo general, inferior en relación a la calidad de la oferta que brindan las escuelas de los sectores de mayores ingresos. Ello conlleva a que los estudiantes procedentes de las familias de menores niveles socioeconómicos y culturales se vean enfrentados a una doble inequidad, por una parte, a causa de la segregación escolar y, por otra, a la baja calidad de la educación que en general reciben.

En la actualidad, se cuenta con abundante información acerca de las causas y los efectos poco deseables que produce la segregación escolar a nivel educativo y social, constituyéndose en un factor gravitante de los resultados educativos, así como también para la convivencia e integración social. En este contexto, sin pecar de ingenuos, queremos pensar que los esfuerzos que muchos países vienen haciendo a través de sus reformas educativas, por avanzar hacia sistemas educativos y escuelas más inclusivas, están en la dirección correcta, en términos de ayudar a contener o atenuar los niveles de segregación escolar que afectan a los países latinoamericanos. Sin embargo, más allá de la retórica y las buenas intenciones es necesario conocer cuál es la magnitud del problema de la segregación escolar por condiciones socioeconómicas en la Región y cuál ha sido su comportamiento en los últimos 15 años.

Específicamente, esta investigación busca conocer la evolución de la segregación escolar por origen socioeconómico en Educación Secundaria Inferior en algunos países de América Latina, realizando una explotación especial de todas las ediciones de PISA disponibles hasta la actualidad: 2000, 2003, 2006., 2009, 2012 y 2015.

\section{MARCO TEÓRICO}

Es posible distinguir dos dimensiones de segregación escolar que llevan a dos concepciones diferentes de la misma: por un lado, la dimensión de uniformidad (evenness) a partir de la cual segregación escolar es la distribución desigual de los estudiantes en las escuelas en función de sus características personales o sociales o condiciones (Dupriez 2010; Frankel y Volij 2011; Murillo 2016). De otra parte, a través de la dimensión de exposición (exposure), se entiende como segregación escolar la probabilidad de que un estudiante de determinada característica se encuentre con otro en su escuela (Allen y Vignoles 2006; Johnston, Wilson 
y Burgess 2004). De esta forma, en función de la definición utilizada, será diferente la forma de estimarla y, con ello, diferentes los resultados obtenidos.

Para estimar la segregación escolar en su dimensión de uniformidad los índices más habituales son el índice de Duncan (Duncan y Duncan 1955), el de la Raíz Cuadrada de Hutchens (Hutchens 2001, 2004) y el índice de Gorard (Gorard 2000), cada uno de ellos con sus propias características (Murillo 2016). Para calcular la segregación escolar en su dimensión de exposición, el más habitual es el índice de Aislamiento (Lieberson 1981), aunque también puede usarse el de Interacción (Morgan 1983). Como más adelante se justificará, en esta investigación se considera la segregación desde ambas dimensiones y se utilizará el índice de Gorard y el de Aislamiento.

La segregación escolar también será diferente en función de las características de los estudiantes que se consideren. A lo largo de los 60 años de investigación sobre esta temática se ha abordado la segregación étnica apelando a la diversidad étnico-cultural de los estudiantes (Reardon y Owens 2014; Stroub y Richards 2013), la segregación escolar por origen nacional de los estudiantes en función de si han nacido no en el país donde estudian o no (Entorf y Lauk 2008; Karsten 2010; Murillo, Martínez-Garrido y Belavi 2017), y la segregación escolar por nivel socioeconómico de las familias de los estudiantes (p. ej., Dupriez 2010; Gorard y Smith 2004; Owens, Reardon y Jencks 2016). La primera desarrollada en gran medida en Estados Unidos desde mediados de los años 50, pero con menos fuerza en la actualidad, la segunda muy extendida en Europa, y la tercera con incidencia en todo el mundo desde los años 80. En esta investigación nos centraremos en la segregación escolar por nivel socioeconómico de las familias.

Las causas de la segregación escolar son múltiples y complejas, destacando, en primer lugar, los factores contextuales especialmente la segregación residencial. Según la misma la concentración en barrios de familias de análogas características sociodemográficas, algo muy habitual en América Latina, genera que los estudiantes que asisten a las escuelas localizadas en esos barrios presenten similares características sociodemográficas (Taylor y Gorard 2001). Pero también hay algunas decisiones de política pública que favorecen una mayor segregación escolar. Una de ellas son las políticas de fomento de mecanismos de cuasi-mercado escolar que potencian la competitividad entre escuelas, publicando rankings de escuelas según resultados de aprendizaje y favoreciendo la libertad de elección, incluso con políticas de financiamiento al puesto escolar, las que sin duda generan una alta segregación (Elacqua 2012; Saporito 2003; Valenzuela, Bellei y De los Ríos 2014). También políticas de fomento de educación privada con exigencias de cumplimiento de criterios de acceso o permanencia selectivos. (Burgess, Wilson y Lupton 2005; Karsten et al. 2006; Nechyba 2006). Estas prácticas contribuyen a que las escuelas presionadas por conseguir mejores resultados, prefieran a los estudiantes de mejores condiciones socioeconómicas y culturales, que suelen tener mejores niveles de asistencia, permanencia y logros de aprendizaje y, tiendan a discriminar a aquellos más "costosos" de educar, los estudiantes con menores recursos sociales y culturales (Valenzuela, Bellei y De los Ríos 2010).

Las consecuencias de la segregación escolar son muchas y no siempre bien conocidas ni estudiadas. La segregación escolar incide negativamente en el aprendizaje de los estudiantes (Agirdag, Van Avermaet y Van Houtte 2013; Rumberger y Palardy 2005) y en su bienestar (McArdle y Acevedo-Garcia 2017), .empobrece el rol cohesionador de la escuela como espacio formativo para la socialización, la convivencia entre personas 
diferentes y la integración social (Ayscue y Orfield 2016), contribuyendo a la generación de fenómenos de discriminación, exclusión y desintegración en la sociedad (Orfield 2001), que terminan afectando a todos.

Por otro lado, daña el demostrado efecto de los iguales (peer effect), derivado de la convivencia e interacción entre los alumnos. Los estudios tienden a coincidir en que escuelas y clases menos segregadas benefician a todos los estudiantes y comparativamente más a los alumnos en situación de mayor vulnerabilidad, ya sea por condiciones socioeconómicas, raciales, o académicas (Harker y Tymms 2004; Kao y Thompson 2003; Valenzuela, Bellei y De los Ríos 2010; Van Ewijk y Sleegers 2010a, 2000b; Zimmerman 2003). Dado que los estudiantes de peor condición socioeconómica proceden de familias con menores recursos socioculturales, estos se benefician de la interacción con compañeros de mayores recursos. La segregación, por tanto, incide claramente en el desarrollo académico de los estudiantes, perjudicando a los de niveles socioeconómicos más bajos. Este fenómeno también se produce porque en las escuelas con una mayor concentración de estudiantes provenientes de familias de nivel socioeconómico bajo, los procesos y recursos educativos tienden a ser de peor calidad. Existen evidencias de las mayores dificultades que dichas escuelas tienen, por ejemplo, para reclutar y mantener un cuerpo docente cualificado, o para conseguir mejores recursos educativos (Valenzuela, Bellei y De los Ríos 2010).

Actualmente se cuenta con evidencias de la segregación por nivel socioeconómico en las escuelas de prácticamente todo el mundo: Países Bajos (Gramberg 1998), España (Benito y Gonzàlez 2007; Murillo y Martínez-Garrido 2018), Francia (Oberti 2012), Inglaterra (Goldstein y Noden 2003; Gorard 1998; Gorard y Fitz 2000; Gorard y Smith 2004; Harris 2012; Noden 2000), Suecia (Böhlmark et al. 2016), Pakistán (Siddiqui 2017), o China (Zhou, Cai y Wang 2016), por poner algunos ejemplos. También se han desarrollado en América Latina, básicamente desde al año 2000, excelentes trabajos que nos aporten una imagen de su situación. Así, se destacan trabajos de Krüger en Argentina, de Bartholo en Brasil, de Bellei, Valenzuela, Valenzuela, Bellei y De Los Rios en Chile, de Duarte, Bos y Moreno en Colombia, de Murillo y Martínez-Garrido en Ecuador, de Backhoff en México, o de Balarin, en Perú (Krüger 2014; Bartholo 2013; Bellei 2013; Valenzuela 2008; Valenzuela, Bellei y De Los Rios 2008; Duarte, Bos y Moreno 2012; Murillo y MartínezGarrido 2017 ; ; Backhoff 2011; Balarin 2016). Además, se cuenta con trabajos que ofrecen una visión conjunta de la segregación escolar por nivel socioeconómico en varios países de la Región de América Latina sirviéndose de los datos de las evaluaciones internacionales como TERCE o PISA, es el caso de los trabajos de Murillo y Martínez-Garrido o Vázquez (Murillo y Martínez-Garrido 2017b; Vázquez 2012).

Sin embargo, son muy pocos los estudios que han analizado la evolución de la segregación escolar por nivel socioeconómico en la América Latina, pese a los efectos negativos que esta tiene tal y como se ha venido argumentando en este trabajo. Entre ellos destacan los realizados en Argentina por Gasparini, Jaume, Serio y Vázquez y Krüger, en Chile por Valenzuela, Bellei y De los Ríos, y los realizados con carácter internacional por Benavides, León y Etesse y Arcidiácono y colaboradores (Gasparini, Jaume, Serio y Vázquez 2011; Krüger 2014; Valenzuela, Bellei y De los Ríos 2008; Benavides, León y Etesse 2014; Arcidiácono y colaboradores 2014).

En Argentina, Gasparini y colaboradores y Krüger analizan la evolución de la segregación escolar por nivel socioeconómico (Gasparini y colaboradores 2011; Krüger 2014). En primer lugar, Leonardo Gasparini, David Jaume, Monserrat Serio y Emmanuel 
Vázquez del Centro de Estudios Distributivos, Laborales y Sociales de la Universidad Nacional de La Plata, utilizan los datos del Instituto Nacional de Estadística y Censos desde 1992 hasta 2009 y realizan el estudio en las escuelas de Educación Primaria y Secundaria del país (Gasparini, Jaume, Serio y Vázquez 2011). Los autores estudian las dos dimensiones de la segregación estimando el índice de Disimilitud y Brecha por Centiles (dimensión de uniformidad) y el índice de Aislamiento (dimensión de exposición) y consideran como grupo minoritario al $20 \%$ de los estudiantes cuyas familias poseen un menor nivel de ingreso per cápita. Los autores señalan que la segregación escolar por nivel socioeconómico en las escuelas de Primaria ha aumentado de 0,24 a 0,34 en los 17 años contemplados. En el caso de las escuelas de Educación Secundaria, se observa un aumento algo más moderado, de 0,20 a 0,27 (valores medidos a través del índice de Disimilitud). Además, los resultados indican que los estudiantes con menor nivel socioeconómico en Secundaria están cada vez más expuestos, el índice de Aislamiento varía de 0,23 en 1992 a 0,43 en 2009.

Natalia Krüger (2014), del Consejo Nacional de Investigaciones Científicas y Técnicas (CONICET) de Argentina, utiliza los datos PISA 2000 y PISA 2009 para estudiar la evolución de la segregación escolar por nivel socioeconómico se las escuelas de Educación Secundaria argentinas en la dimensión de uniformidad y de exposición a partir de la estimación de los índices de Disimilitud, de Hutchens y de Aislamiento (Krüger 2014). La autora utiliza cuatro variables criterio: el estatus ocupacional de los padres, su categoría de empleo, su nivel educativo, y las posesiones en el hogar. Y estima la segregación para seis grupos minoritarios: a) Estatus ocupacional bajo (empleo "cuello azul-baja capacitación”), b) Estatus ocupacional alto (empleo "cuello blanco-alta capacitación”), c) Nivel educativo de los padres bajo (estudios primarios), d) Nivel educativo de los padres alto (estudios terciarios), e) Nivel de posesiones bajo (valor menor al Q1), y f) Nivel de posesiones alto (valor mayor al Q4). Entre sus resultados destaca un aumento del 3\% en la segregación para los estudiantes cuyas familias cuentan con un estatus ocupacional bajo (de 0,36 a 0,39 , valores medidos a través del índice de Disimilitud). O que los estudiantes con menores posesiones en el hogar familiar (estudiantes del Q1) están cada vez más segregados, el valor de la segregación en el año 2000 es 0,41, y de 0,43 en el año 2009 (valores medidos a través del índice de Aislamiento).

En Chile, el estudio realizado entre 1999 y 2006 por Juan Pablo Valenzuela, Cristian Bellei y por Danae de los Ríos (De los Ríos 2008) del Centro de Investigación Avanzada en Educación de la Universidad de Chile, analiza la evolución de la segregación por nivel socioeconómico en las escuelas de Educación Secundaria del país utilizando los datos del SIMCE. Los autores estiman el índice de Disimilitud (dimensión de uniformidad) para el $30 \%$ de los estudiantes cuyas familias cuentan con menor y mayor nivel socioeconómico. Los resultados del estudio determinan que, a lo largo de los siete años de estudio, la segregación en el país ha aumentado más para los estudiantes de bajo nivel socioeconómico (un 8\%) que para los estudiantes de mayor nivel socioeconómico (un 7\%). Así, la segregación en 2006 para el 30\% de los estudiantes con menor nivel socioeconómico es de 0,61 y de 0,57 para los estudiantes con mayor nivel socioeconómico. El estudio de los municipios arroja valores extremos, según los autores el $20 \%$ de los municipios sufren un aumento del $10 \%$ en su nivel de segregación entre los años 1999 y 2006.

Por su parte, las investigaciones que han abordado el estudio de la evolución de la segregación escolar por nivel socioeconómico abarcando varios países de América Latina, 
destacan los realizados por Benavides, León y Etesse y Arcidiácono y sus colaboradores (Benavides, León y Etesse 2014; Arcidiácono y colaboradores 2014). De un lado, los investigadores Martín Benavides, Juan León y Manuel Etesse, del Grupo de Análisis para el Desarrollo (GRADE) en Perú, utilizan los datos de la evaluación internacional PISA de los años 2000 y 2009 para medir la evolución de la segregación por nivel socioeconómico en las escuelas de Educación Secundaria en Argentina, Brasil, Chile, México y Perú (Benavides, León y Etesse 2014). Para alcanzar su objetivo, estos autores estiman el índice de Hutchens que permite ver cuán segregados están los sistemas educativos en cada país, o en qué medida la distribución de la población en términos socioeconómicos se reproduce al interior de las escuelas de los países estudiados (dimensión de uniformidad). El estudio considera como punto de corte para definir el grupo minoritario el primer cuartil, esto es, el $25 \%$ de los estudiantes con un menor nivel socioeconómico. Los resultados indican que en los nueve años del estudio el valor promedio de la segregación en los países estudiados se incrementó de 0,23 a 0,26. Los países en los que más aumenta la segregación son Perú y México. En Perú, la segregación en el año 2000 era 0,18 y aumenta hasta 0,31 en 2009, un aumento algo menor se produce en México que pasa de 0,24 en el año 2000 a 0,27 en 2009.

De otro lado, Malena Arcidiácono, Guillermo Cruces, Leonardo Gasparini, David Jaume, Monserrat Serio y Emmanuel Vázquez, del Centro de Estudios Distributivos, Laborales y Sociales de la Universidad Nacional de La Plata, se sirven de los datos del SEDLAC para estudiar la evolución de la segregación escolar por nivel socioeconómico en las escuelas de Etapa Secundaria en 14 países de América Latina desde el año 1992 hasta 2011 (Arcidiácono, Cruces, Gasparini, Jaume, Serio y Vázquez 2014). Los autores utilizan el índice de Disimilitud y Brecha por Centiles (dimensión de uniformidad) y el índice de Aislamiento (dimensión de exposición), y como punto de corte el primer quintil de la variable criterio ingreso per cápita familiar. Los resultados apuntan que la segregación escolar por nivel socioeconómico en los 19 años estudiados ha aumentado de 0,15 a 0,17 según el índice de Disimilitud, y de 0,25 a 0,30 según el índice de Aislamiento. Cifras excepcionalmente bajas que probablemente se deban al atrevimiento de los autores por utilizar datos que provienen de 23 encuestas y bases de datos (Gorard 2006).

La investigación realizada hasta el momento tan sólo ofrece algunas evidencias en algunos países en momentos puntuales y aunque algunos trabajos han analizado la evolución de la segregación escolar por nivel socioeconómico, éstos se han realizado tan sólo durante la primera década del siglo. Por ello, la presente investigación busca conocer la evolución de la segregación escolar de carácter socioeconómico en América Latina, de modo de poder dimensionarla y conocer sus tendencias habida cuenta de sus efectos en la población escolar y la sociedad, ampliamente descritos en este trabajo.

\section{MÉTODO}

Para alcanzar este objetivo se realizó una explotación especial de los datos de los países de América Latina que participaron en las diferentes ediciones de PISA: 2000, 2003, 2006, 2009, 2012 y 2015. Concretamente se analizaron los datos correspondientes a Argentina, Brasil, Chile, Colombia, Costa Rica, República Dominicana, México, Panamá, Perú y Uruguay. 
La muestra del estudio está conformada, por tanto, por 374.490 estudiantes de 15.500 escuelas ubicadas en los diez países mencionados. Para la selección de la muestra, PISA utiliza un muestreo estratificado en dos etapas. Las unidades de muestreo de la primera etapa son las escuelas con estudiantes de 15 años de edad. Estas se seleccionan sistemáticamente a partir de una lista a nivel nacional completa de todas las escuelas elegibles para PISA, con probabilidad de selección proporcional al número estimado de estudiantes de 15 años elegibles para PISA matriculados en dicha escuela (cuadro 1).

Cuadro 1. Muestra de estudiantes y escuelas participantes en la investigación

\begin{tabular}{|l|c|c|c|c|c|c|c|c|c|c|c|c|}
\hline & \multicolumn{2}{|c|}{2000} & \multicolumn{2}{c|}{2003} & \multicolumn{2}{c|}{2006} & \multicolumn{2}{c|}{2009} & \multicolumn{2}{c|}{2012} & \multicolumn{2}{c|}{2015} \\
\cline { 2 - 15 } & Est. & Esc. & Est. & Esc. & Est. & Esc. & Est. & Esc. & Est. & Esc. & Est. & Esc. \\
\hline Argentina & 2.230 & 156 & - & - & 4.339 & 171 & 4.774 & 199 & 5.908 & 226 & - & - \\
\hline Brasil & 2.717 & 324 & 4.597 & 228 & 9.295 & 625 & 20.127 & 947 & 19.204 & 839 & 23.141 & 841 \\
\hline Chile & 2.721 & 179 & - & - & 5.233 & 173 & 5.669 & 200 & 6.856 & 221 & 7.053 & 227 \\
\hline Colombia & - & - & - & - & 4.478 & 165 & 7.921 & 275 & 9.073 & 352 & 11.795 & 372 \\
\hline Costa Rica & - & - & - & - & - & - & 4.578 & 181 & 4.602 & 193 & 6.866 & 205 \\
\hline R. Dominicana & - & - & - & - & - & - & - & - & - & - & 4.740 & 194 \\
\hline México & 2.567 & 183 & 29.983 & 1.124 & 30.971 & 1.140 & 38.250 & 1535 & 33.806 & 1471 & 7.568 & 275 \\
\hline Panamá & - & - & - & - & - & - & 3.969 & 188 & - & - & - & - \\
\hline Perú & 2.460 & 177 & - & - & - & - & 5.985 & 240 & 6.035 & 240 & 6.971 & 281 \\
\hline Uruguay & - & - & 5.835 & 243 & 4.839 & 278 & 5.957 & 232 & 5.315 & 180 & 6.062 & 220 \\
\hline
\end{tabular}

Fuente. Elaboración propia a partir de PISA 2000, 2003, 2006, 2009, 2012 y 2015.

La variable utilizada para estimar la segregación es el "Nivel Socioeconómico y Cultural de las familias de los estudiantes" (ESCS, Index of economic, social and cultural status) estimada por PISA y publicada en 2016 para todas las ediciones. Este constructo fue estimado por PISA a partir de la información de las siguientes variables: Índice Socioeconómico Internacional de Situación Laboral (ISEI), el nivel educativo más alto de los padres del estudiante, convertidos en años de escolaridad; el índice de la riqueza familiar, el índice de recursos educativos del hogar y el índice de posesiones relacionadas con la cultura en el domicilio familiar. Esta variable supone una corrección de los datos que aparecen en las bases de datos de las diferentes ediciones, de tal forma que es comparable entre ediciones.

Para alcanzar los objetivos del estudio se utilizaron dos índices diferentes, el índice de segregación de Gorard para estimar la dimensión de uniformidad, y el índice de Aislamiento para calcular la dimensión de exposición. 
El índice de segregación de Gorard, o índice G (Gorard 2000; Gorard y Taylor 2002), es una variación del popular índice de Disimilitud (Duncan y Duncan 1955), pero supera la limitación de estar influido por el tamaño del grupo minoritario. Este procedimiento estima la magnitud de la segregación a través del cálculo de la proporción de sujetos del grupo minoritario que deberían cambiar de unidad educativa para que exista una distribución homogénea de los mismos entre las distintas escuelas, estima el nivel de segregación considerando la diferencia entre la proporción del grupo minoritario y la proporción de todos los miembros del grupo.

$$
\text { El procedimiento de cálculo es: } G=\frac{1}{2} \sum_{i=1}^{k}\left|\frac{X_{1 i}}{X_{1}}-\frac{T_{i}}{T}\right|
$$

Donde, para país, x 1 i representa el número de alumnos del grupo minoritario en la escuela i, X1 es el número total de estudiantes del grupo minoritario en todas las escuelas del país, Ti es el número total de alumnos en la escuela i, y T, número total de alumnos en cada país.

Con vistas a ofrecer una imagen completa de la segregación escolar por nivel socioeconómico, y dada la importancia de la elección del grupo minoritario (Murillo 2016) se han establecido cuatro puntos de corte para conformar dicho grupo:

- El 10\% de los estudiantes con familias de menos nivel socioeconómico y cultural (P10)

- El $25 \%$ de los estudiantes con familias de menos nivel socioeconómico y cultural (Q1)

- El 25\% de los estudiantes con familias de mayor nivel socio-económico y cultural (Q4)

- $\quad$ El $10 \%$ de los estudiantes con familias de mayor nivel socioeconómico y cultural (P90)

El segundo índice utilizado en esta investigación es el de Aislamiento (A), dado que es el más habitual para estimar la dimensión de exposición de la segregación escolar. Fue propuesto por Lieberson (Lieberson 1981) y se interpreta como la probabilidad de que un estudiante del grupo minoritario se encuentre en la escuela con otro miembro de su grupo. Es decir, cuanto mayor sea el aislamiento del grupo, menor será su exposición a los miembros del resto de grupos. Al captar el grado de contacto potencial de los estudiantes de nivel socioeconómico bajo con otros alumnos de su mismo grupo, es un índice que está afectado por la participación relativa de este grupo en la población total de estudiantes (Echenique y Fryer 2007). Su fórmula es:

$$
A=\sum_{i=1}^{k} \frac{x_{1 i}}{X_{1}} \frac{x_{1 i}}{T_{i}}
$$

Donde, $\mathrm{x}_{1 \mathrm{i}}$ representa el número de alumnos del grupo minoritario en la escuela $\mathrm{i}, \mathrm{X}_{1}$ es el número total de estudiantes minoritarios, respectivamente, en todas las escuelas del país, y $\mathrm{T}_{\mathrm{i}}$ es el número total de alumnos en la escuela $\mathrm{i}$.

Al igual que sucedía con el índice de Gorard, se estimará el índice de Aislamiento para los cuatro puntos de corte definidos: P10, Q1, Q4 y P90. 


\section{RESULTADOS}

En este apartado se muestran, en primer lugar, los resultados de la estimación de la evolución de la magnitud de la segregación por nivel socioeconómico en su dimensión de uniformidad, es decir utilizando en índice de Gorard. A continuación, se presentan los resultados de la dimensión de segregación como exposición; es decir, mediante el índice de Aislamiento.

\subsection{SEGREGACIÓN ESCOLAR COMO UNIFORMIDAD}

No es fácil aportar una imagen general de la evolución de la segregación escolar por nivel socioeconómico en los diez países analizados de América Latina. Dos razones para ello: en primer lugar, porque no todos los países participaron en las seis ediciones, pero también porque el comportamiento de cada país es diferente en los años analizados, y dentro de cada país la evolución para cada grupo minoritario es distinto.

En todo caso, señalaremos algunas regularidades (cuadro 2):

1. En todos los países y en todo momento la segregación escolar de los estudiantes de familias con mayor nivel socioeconómico es mayor que hacia los de menor nivel.

2. La tendencia general es a una disminución de la segregación escolar. Aunque en Colombia y en Perú aumenta la segregación promedio.

3. Sin embargo, la segregación escolar para los estudiantes con familias de menos nivel socioeconómico y cultural (tanto P10 como Q1), la tendencia es al alza. Así sólo disminuye en Colombia, Costa Rica y Uruguay para el P10, y en Brasil y Uruguay para el Q1.

4. Para los estudiantes de familias con mayor nivel socioeconómico, por el contrario, la tendencia es una disminución de la segregación escolar: para el P90 bajan todos los países menos Uruguay, y para el Q4 excepto Colombia, Perú y Uruguay que suben, el resto muestra una tendencia a la baja.

Cuadro 2. Evolución de la segregación escolar por nivel socioeconómico en Educación Secundaria Inferior en América Latina. Índice de Gorard para el 10\% y 25\% de los estudiantes con familias de menor y de mayor nivel socioeconómico y cultural

\begin{tabular}{|l|c|c|c|c|c|c|c|}
\hline & & $\mathbf{2 0 0 0}$ & $\mathbf{2 0 0 3}$ & $\mathbf{2 0 0 6}$ & $\mathbf{2 0 0 9}$ & $\mathbf{2 0 1 2}$ & $\mathbf{2 0 1 5}$ \\
\hline \multirow{4}{*}{ Argentina } & $\mathbf{P 1 0}$ & 0,4673 & - & 0,4643 & 0,4806 & 0,4623 & . \\
\cline { 2 - 8 } & $\mathbf{Q 1}$ & 0,3478 & - & 0,3470 & 0,322 & 0,3437 & . \\
\cline { 2 - 9 } & $\mathbf{Q 4}$ & 0,4021 & - & 0,3681 & 0,3948 & 0,3620 & $\cdot$ \\
\cline { 2 - 9 } & $\mathbf{P 9 0}$ & 0,5770 & - & 0,5158 & 0,5782 & 0,4977 &. \\
\hline \multirow{5}{*}{ Brasil } & $\mathbf{P 1 0}$ & 0,4799 & 0,4840 & 0,5488 & 0,5254 & 0,5089 & 0,4816 \\
\cline { 2 - 9 } & $\mathbf{Q 1}$ & 0,3661 & 0,3313 & 0,3653 & 0,3589 & 0,3477 & 0,3274 \\
\cline { 2 - 9 } & $\mathbf{Q 4}$ & 0,4185 & 0,3947 & 0,3774 & 0,3421 & 0,3649 & 0,3420 \\
\cline { 2 - 8 } & $\mathbf{P 9 0}$ & 0,5825 & 0,6186 & 0,6008 & 0,5459 & 0,5561 & 0,5126 \\
\hline
\end{tabular}


Estudios Pedagógicos XLIV, $\mathrm{N}^{\circ}$ 1: 157-179, 2018

EVOLUCIÓN DE LA SEGREGACIÓN SOCIOECONÓMICA DE LAS ESCUELAS DE AMÉRICA LATINA

\begin{tabular}{|c|c|c|c|c|c|c|c|}
\hline \multirow{4}{*}{ Chile } & $\mathbf{P 1 0}$ & 0,4827 & - & 0,5369 & 0,5073 & 0,5498 & 0,5175 \\
\hline & Q1 & 0,3630 & - & 0,3945 & 0,3722 & 0,3968 & 0,3719 \\
\hline & Q4 & 0,4439 & - & 0,4621 & 0,4322 & 0,4509 & 0,4018 \\
\hline & P90 & 0,6034 & - & 0,6223 & 0,6009 & 0,6312 & 0,5766 \\
\hline \multirow{4}{*}{ Colombia } & $\mathbf{P} 10$ & - & - & 0,4940 & 0,4773 & 0,5236 & 0,4679 \\
\hline & Q1 & - & - & 0,3089 & 0,3601 & 0,3582 & 0,3511 \\
\hline & Q4 & - & - & 0,3402 & 0,3534 & 0,3392 & 0,3837 \\
\hline & P90 & - & - & 0,5032 & 0,5005 & 0,4901 & 0,5941 \\
\hline \multirow{4}{*}{ Costa Rica } & P10 & - & - & - & 0,4577 & 0,4754 & 0,4102 \\
\hline & Q1 & - & - & - & 0,3085 & 0,3258 & 0,3105 \\
\hline & Q4 & - & - & - & 0,3807 & 0,3593 & 0,3655 \\
\hline & P90 & - & - & - & 0,5597 & 0,5499 & 0,5341 \\
\hline \multirow{4}{*}{ Rep. Dominicana } & $\mathbf{P 1 0}$ & - & - & - & & & 0,4195 \\
\hline & Q1 & & & & & & 0,3238 \\
\hline & Q4 & & & & & & 0,3306 \\
\hline & P90 & & & & & & 0,5024 \\
\hline \multirow{4}{*}{ México } & P10 & 0,4837 & 0,4914 & 0,5636 & 0,5521 & 0,5563 & 0,5401 \\
\hline & Q1 & 0,3727 & 0,3736 & 0,4366 & 0,3953 & 0,4078 & 0,3940 \\
\hline & Q4 & 0,4200 & 0,3641 & 0,3772 & 0,3709 & 0,3738 & 0,3533 \\
\hline & P90 & 0,5728 & 0,5312 & 0,5428 & 0,5381 & 0,5346 & 0,5239 \\
\hline \multirow{4}{*}{ Panamá } & P10 & - & - & - & 0,4716 & & \\
\hline & Q1 & & & & 0,3641 & & \\
\hline & Q4 & & & & 0,4595 & & \\
\hline & P90 & & & & 0,5322 & & \\
\hline \multirow{4}{*}{ Perú } & P10 & 0,4397 & - & - & 0,5340 & 0,5529 & 0,5954 \\
\hline & Q1 & 0,344 & - & - & 0,4234 & 0,4343 & 0,4754 \\
\hline & Q4 & 0,359 & - & - & 0,3788 & 0,3827 & 0,4053 \\
\hline & P90 & 0,531 & - & - & 0,564 & 0,5261 & 0,5472 \\
\hline \multirow{4}{*}{ Uruguay } & P10 & - & 0,4466 & 0,4836 & 0,4001 & 0,4083 & 0,3879 \\
\hline & Q1 & - & 0,3156 & 0,3457 & 0,2921 & 0,3005 & 0,2999 \\
\hline & Q4 & - & 0,3498 & 0,3908 & 0,3180 & 0,3886 & 0,3640 \\
\hline & P90 & - & 0,4800 & 0,5581 & 0,4319 & 0,5944 & 0,5445 \\
\hline
\end{tabular}

Fuente. Elaboración propia a partir de PISA 2000, 2003, 2006, 2009, 2012 y 2015. 
Un análisis de cada uno de los diez países examinados aporta una información más rica y completa.

De Argentina solo se cuenta información de cuatro de las seis ediciones de PISA, faltando los datos del 2003 y del 2015. En comparación con los otros estudiados, se puede afirmar que es uno de los países con una menor segregación escolar, por detrás de Uruguay y, depende del año, a la altura de Costa Rica o República Dominicana. El análisis de la evolución de la magnitud de la segregación en las cuatro ediciones con datos muestra una disminución para el $25 \%$ y el $10 \%$ de los estudiantes con familias de mayor nivel socioeconómico (Q4 y P90), y un comportamiento prácticamente plano para los estudiantes con familias de menor nivel socioeconómico (P10 y Q1). Con mayor precisión:

- Para el P10 se mantiene en torno a 0,46 en estos años, con mínimas variaciones.

- Para el Q1 el comportamiento es también muy estable, en torno a 0,34 desde 2000 a 2012.

- Para el Q4, se observa una disminución de 0,40 a 0,36, también con relativa estabilidad.

- Para el P90. por último, la disminución llega a las 8 centésimas, de 0,58 en 2000 a 0,50 en 2012.

Gráfico 1. Evolución de la segregación escolar por nivel socioeconómico en Argentina. Índice de Gorard para el P10, Q1, Q4 y P90 como grupo minoritario

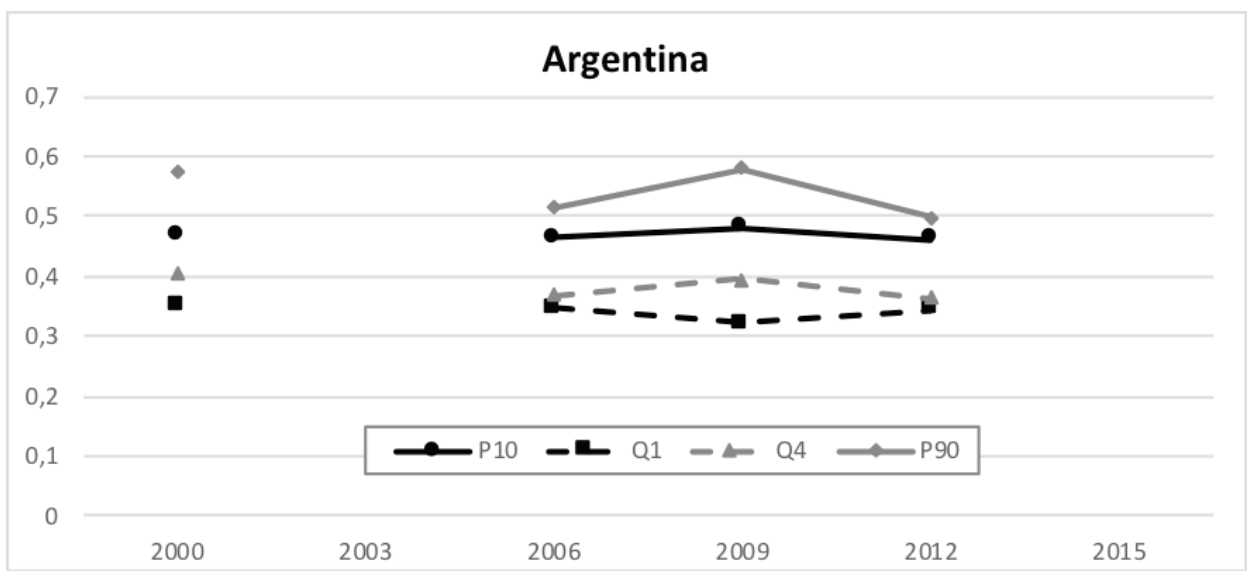

Fuente: Elaboración propia a partir de PISA 2000, 2003, 2006, 2009, 2012 y 2015.

En los 15 años analizados, Brasil mantiene una tendencia a la baja en la segregación escolar por nivel socioeconómico para tres de los cuatro grupos minoritarios estudiados. En el único que no se observa esa evolución es para el 10\% de los estudiantes con familias de menor nivel socioeconómico. En ese caso, tuvo un fuerte incremento del 2000 al 2006 y de ahí una progresiva disminución, con lo que el cambio es inapreciable, manteniéndose en el 0,48 . 
Para el resto de los colectivos analizados:

- $\quad$ Para el Q1, la pendiente es de: -0,0043 (R2=0,23)

- $\quad$ Para el Q4, la pendiente 0,0144 (R2=0,81)

- $\quad$ Para el P90, la pendiente es de $-0,0169(\mathrm{R} 2=0,66)$

De esta forma, se puede concluir que Brasil ha bajado en su segregación para los estudiantes con familias de mayor nivel socioeconómico y cultural. Para los de mayor, la bajada es inexistente o muy baja, con fuertes altibajos. Esas disminuciones han hecho que Brasil sea el tercer país menos segregado entre los estudiados.

Gráfico 2. Evolución de la segregación escolar por nivel socioeconómico en Brasil. Índice de Gorard para el P10, Q1, Q4 y P90 como grupo minoritario

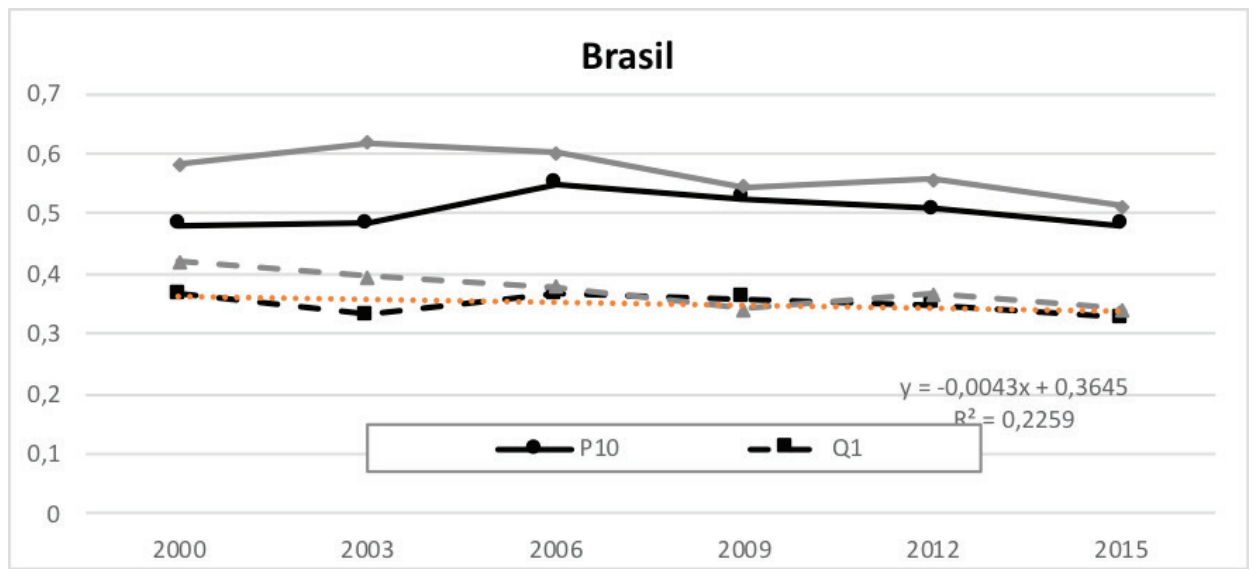

Fuente: Elaboración propia a partir de PISA 2000, 20013, 2006, 2009, 2012 y 2015.

Los perfiles evolutivos de la segregación escolar por nivel socioeconómico en Chile no muestran una tendencia clara, observándose grandes saltos para los distintos momentos analizados. En todo caso, hay grandes diferencias en función del colectivo analizado:

- Para el $10 \%$ de los estudiantes con familias de menos nivel socioeconómico y cultural (P10), la diferencia entre la primera medición (2000) y la última (2015) muestra una moderada subida de más 3 centésimas en el índice de Gorard. Sin embargo, no se ve una tendencia clara.

- Para el 25\% de los estudiantes con familias de menor nivel socioeconómico (Q1), ocurre algo parecido, pero en este caso la segregación aumenta ligeramente en estos 15 años. En todo caso no se observa un cambio importante.

- Para el 25\% de los estudiantes con familias de mayor nivel (Q4), sin embargo, la disminución de la segregación es clara, de más de 4 centésimas y con una tendencia de disminución más estable en los últimos años. 
- Por último, para el $10 \%$ de los estudiantes con familias de mayor nivel socioeconómico (P90), la disminución es de casi 4 centésimas entre la primera medida y la última, pero de nuevo con bastantes oscilaciones.

Como conclusión, Chile no tiene una tendencia clara, pero la comparación entre los 15 años analizados lleva a pensar en un aumento de la segregación para los estudiantes de menos nivel socioeconómico y de disminución para los de mayor nivel.

Gráfico 3. Evolución de la segregación escolar por nivel socioeconómico en Chile. Índice de Gorard para el P10, Q1, Q4 y P90 como grupo minoritario

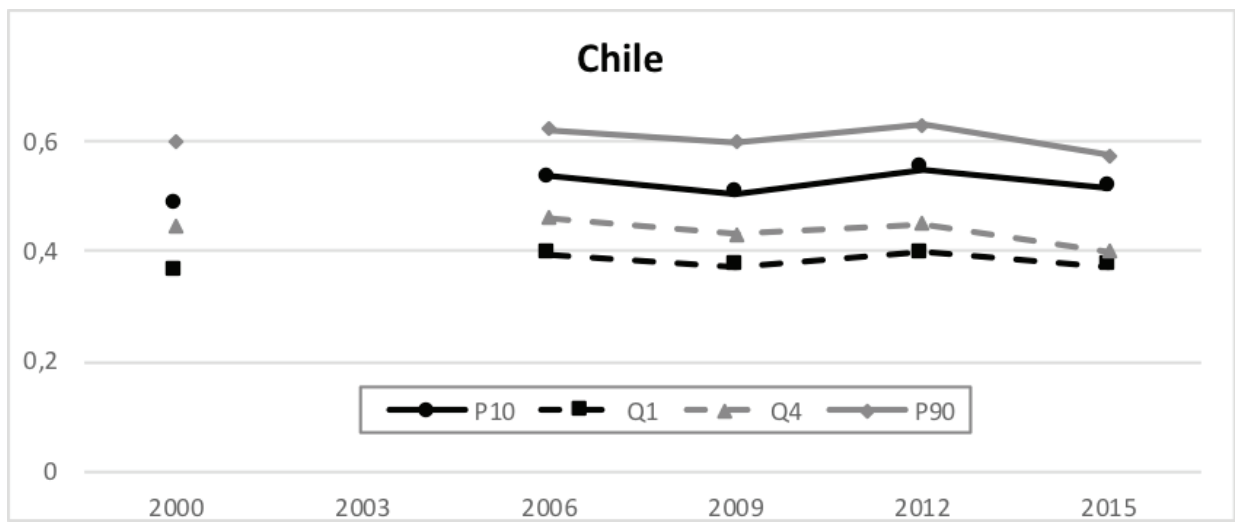

Fuente: Elaboración propia a partir de PISA 2000, 20013, 2006, 2009, 2012 y 2015.

Colombia es uno de los países en los que más aumenta la segregación escolar por nivel socioeconómico de la Región en los años analizados. Efectivamente, aunque solo hay datos de las últimas cuatro ediciones de PISA, el incremento en la segregación es claro y sostenidos para tres de los cuatro colectivos analizados. Solo se observa una mínima disminución de menos de tres centésimas para el $10 \%$ de los estudiantes con familias de menor nivel socioeconómico. Para el Q1, sin embargo, entre 2006 y 2015 se produce un incremento de casi 5 centésimas y una pendiente de 0,013, para el Q4 las cifras son parecidas, un incremento de más de 4 centésimas y la misma pendiente. El incremento más importante es, sin embargo, para el $10 \%$ de los estudiantes con familias de mayor nivel, en ese caso el incremento casi llega a 10 centésimas y una pendiente que duplica las anteriores, de 0,026 . 
Gráfico 4. Evolución de la segregación escolar por nivel socioeconómico en Colombia. Índice de Gorard para el P10, Q1, Q4 y P90 como grupo minoritario

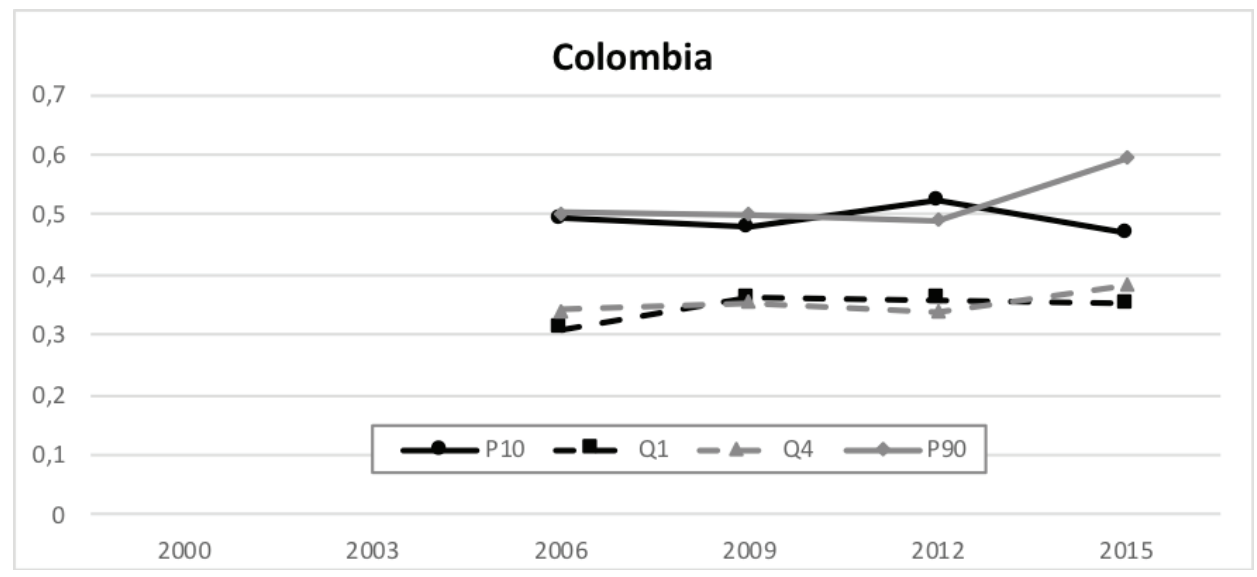

Fuente: Elaboración propia a partir de PISA 2000, 2003, 2006, 2009, 2012 y 2015.

Costa Rica solo participó en las tres últimas ediciones de PISA (PISA 2009, 2012 y 2015). A pesar de la escasa información que se tiene, la tendencia a una menor segregación escolar es clara, con la única excepción de la correspondiente al 25\% de los estudiantes con familias de menor nivel socioeconómico, que apenas sufre alteraciones. Efectivamente para el P10 en esos 6 años estudiados la segregación baja de 0,46 a 0,41, para el q4 la disminución es menor, del 0,38 a 0,37; y para el P90 de 0,56 a 0,53. Todo ello le refuerza como uno de los países de la región con una menor segregación escolar.

Gráfico 5. Evolución de la segregación escolar por nivel socioeconómico en Costa Rica. Índice de Gorard para el P10, Q1, Q4 y P90 como grupo minoritario

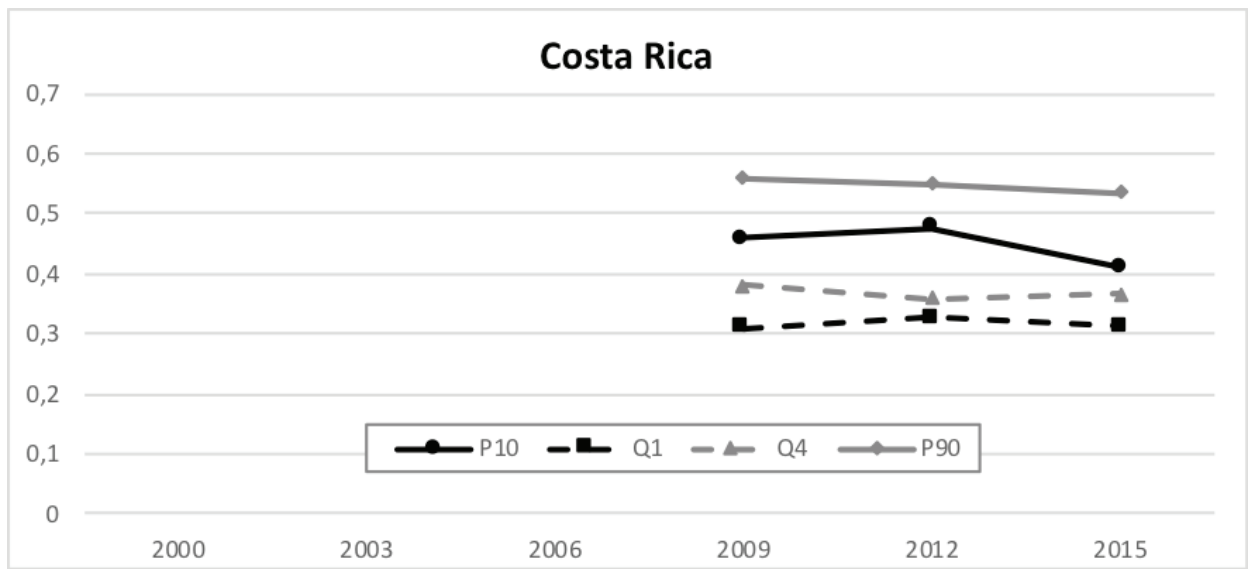

Fuente: Elaboración propia a partir de PISA 2000, 20013, 2006, 2009, 2012 y 2015. 
México es, junto a Brasil, uno de los dos únicos países de América Latina que participaron en todas las ediciones de PISA, de tal forma que se tienen datos completos de su evolución desde 2000 hasta 2015. Con esta rica información, se observa (gráfico 5), una evolución de la magnitud de la segregación escolar al alza para los estudiantes con familias de menor nivel socioeconómico y de disminución para los de mayor nivel. Efectivamente, para el P10 y el Q1, el incremento es de 0.06 y 0,02 respectivamente, y para Q4 y P90 la disminución es de 0,07 y 0,05 respectivamente.

Gráfico 6. Evolución de la segregación escolar por nivel socioeconómico en México. Índice de Gorard para el P10, Q1, Q4 y P90 como grupo minoritario

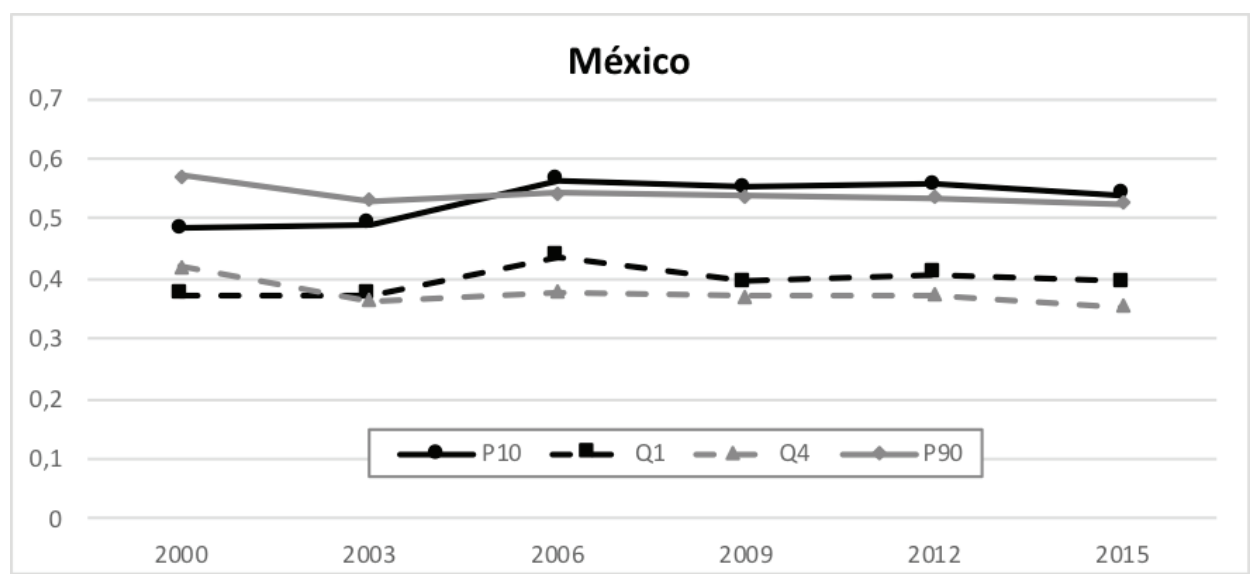

Fuente: Elaboración propia a partir de PISA 2000, 20013, 2006, 2009, 2012 y 2015.

Perú es el país de entre los diez analizados que presenta unas mayores tasas de segregación escolar por nivel socioeconómico. Junto a ello, lo más llamativo es que también es el país que tiene una tendencia al alza en sus niveles de segregación para todos los cuatro grupos minoritarios analizados, pero especialmente para los estudiantes con familias de menos nivel socioeconómico. Efectivamente, entre 2000 y 2015. La segregación para el P10 aumenta en 0,16, para Q1 en 0,13, para Q4 en 0.04 y para P90 en 0,02. 
Gráfico 7. Evolución de la segregación escolar por nivel socioeconómico en Perú. Índice de Gorard para el P10, Q1, Q4 y P90 como grupo minoritario

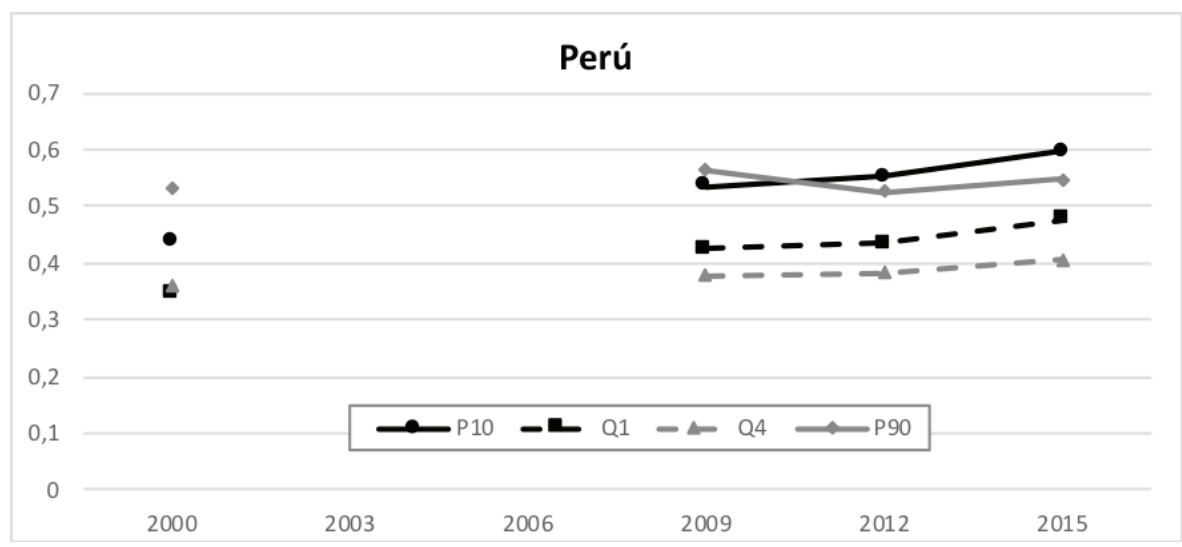

Fuente: Elaboración propia a partir de PISA 2000, 20013, 2006, 2009, 2012 y 2015.

Uruguay, por último, es el país que sistemáticamente ofrece unos menores niveles de segregación escolar promedio de los países de América Latina estudiados. Su comportamiento, sin embargo, también es especial, dado que es uno de los pocos países cuya segregación paja para los grupos minoritarios de estudiantes con familias de menor nivel socioeconómico (0.06 para P10 y 0,02 para Q1) y sube para los de mayor nivel $(0,01$ para Q4 y 0,06 para P90). De esta forma se refuerza su elemento característico de tener una muy baja segregación para los estudiantes con familias de menor nivel socioeconómico y muy alta para los de mayor nivel.

Gráfico 8. Evolución de la segregación escolar por nivel socioeconómico en Uruguay. Índice de Gorard para el P10, Q1, Q4 y P90 como grupo minoritario

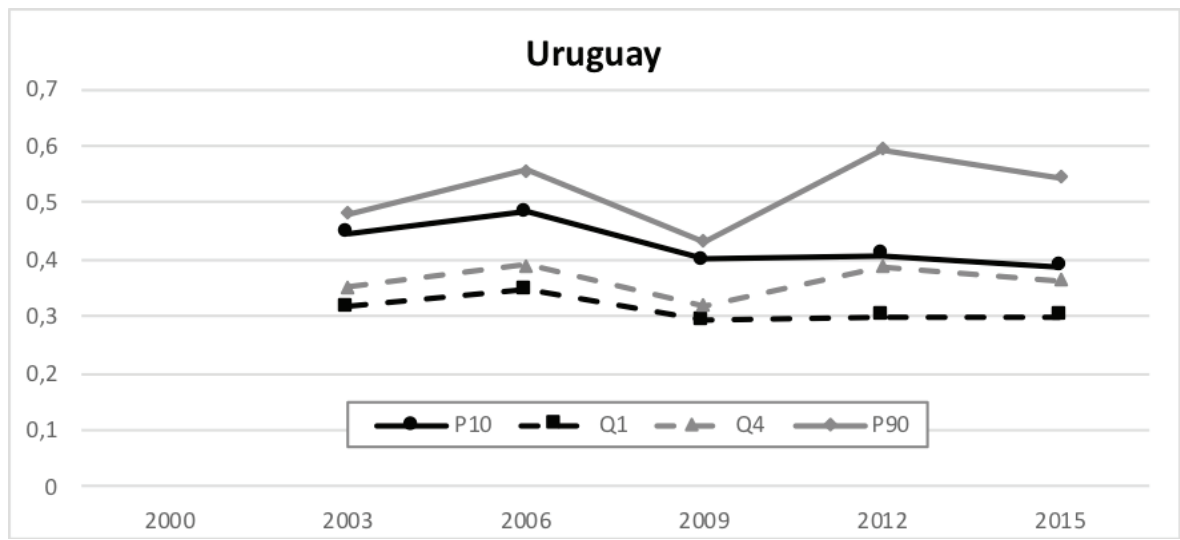

Fuente: Elaboración propia a partir de PISA 2000, 20013, 2006, 2009, 2012 y 2015. 
Además, hay dos países de América Latina que, al participar únicamente en una edición de PISA, no se poseen datos de la evolución de la segregación: República Dominicana y Panamá. La República Dominicana solo participó en la última edición de PISA, y sus índices de segregación le hacen estar entre los países menos segregados de los estudiados, especialmente para los estudiantes con familias de menor nivel socioeconómico. Efectivamente para el P10 y el Q1 es el tercer país con una menor segregación de entre los 10, y en el Q4 y el P90 es el país con un sistema educativo menos segregado. En todo caso, sus cifras son muy altas $(0,42,0,31,0,33$ y 0,50 para cada uno de los cuatro colectivos y en ese orden), lo que indica que aún hay mucho camino que recorrer para mejorar este indicador de desigualdad escolar.

Panamá, por último, solo participó en la edición de PISA de 2009, por lo que a la escasa información que se tiene hay que añadir su desactualización. En todo caso, solo comentar que mientras que para el P10 y el Q1 está en unos valores promedios de los países analizados, para el Q4 era el país más segregado en 2009.

\subsection{SEGREGACIÓN ESCOLAR COMO EXPOSICIÓN}

Una imagen complementaria se obtiene del análisis de la segregación escolar en su dimensión de exposición. Según esta dimensión la segregación escolar es la probabilidad de que un estudiante de un grupo minoritario se encuentre con alguien de su misma situación. Se estima a través del índice de Aislamiento. En este caso y frente a lo que ocurría con el índice de Gorard, le, cuanto más pequeño sea el grupo minoritario menos probabilidad habrá de que se produzca ese encuentro; es decir las estimaciones serán más bajas para el P10 y P90 que para el Q1 y Q4.

En este caso, y de forma similar a lo estudiado con el índice de Gorard, la segregación escolar promedio baja en la mayoría de los países en los años estudiados. Tan solo Colombia y Perú se escapan a esta dinámica subiendo su segregación, pero con muchos matices en cada país (cuadro 3):

- La segregación escolar sube en Argentina y Brasil para el 10\% de los estudiantes con familias con menor nivel socioeconómico y baja para los otros tres grupos minoritarios considerados. En Colombia el fenómeno es justo a la inversa: baja para P10 y sube para los otros tres grupos.

- En Chile y México la segregación escolar sube para los estudiantes con familias de menor nivel socioeconómico (P10 y Q1) y bajan para las de mayor (Q4 y P90). Uruguay, por el contrario, baja para el P10 y el Q1 y sube para el Q4 y P90.

- Costa Rica baja para los cuatro colectivos. Y Perú sube en su segregación escolar para los cuatro grupos minoritarios. 
Cuadro 3. Evolución de la segregación escolar por nivel socioeconómico en Educación Secundaria Inferior en América Latina. Índice de Aislamiento para el 10\% y 25\% de los estudiantes con familias de menor y de mayor nivel socioeconómico y cultural

\begin{tabular}{|l|c|c|c|c|c|c|c|}
\hline & & $\mathbf{2 0 0 0}$ & $\mathbf{2 0 0 3}$ & $\mathbf{2 0 0 6}$ & $\mathbf{2 0 0 9}$ & $\mathbf{2 0 1 2}$ & $\mathbf{2 0 1 5}$ \\
\hline \multirow{5}{*}{ Argentina } & $\mathbf{P 1 0}$ & 0,2425 & - & 0,2634 & 0,2716 & 0,2486 & - \\
\cline { 2 - 9 } & $\mathbf{Q 1}$ & 0,4198 & - & 0,4256 & 0,4130 & 0,4104 & - \\
\cline { 2 - 9 } & $\mathbf{Q 4}$ & 0,5056 & - & 0,4654 & 0,5015 & 0,4353 & - \\
\cline { 2 - 9 } & $\mathbf{P 9 0}$ & 0,3780 & - & 0,3149 & 0,4071 & 0,2649 & - \\
\hline \multirow{5}{*}{ Brasil } & $\mathbf{P 1 0}$ & 0,2505 & 0,2636 & 0,3426 & 0,3095 & 0,2986 & 0,2693 \\
\cline { 2 - 9 } & $\mathbf{Q 1}$ & 0,4346 & 0,4203 & 0,4568 & 0,4416 & 0,4386 & 0,4139 \\
\cline { 2 - 9 } & $\mathbf{Q 4}$ & 0,5332 & 0,5148 & 0,4989 & 0,4702 & 0,4873 & 0,4619 \\
\cline { 2 - 9 } & $\mathbf{P 9 0}$ & 0,5825 & 0,6186 & 0,6008 & 0,5459 & 0,5561 & 0,5126 \\
\hline \multirow{5}{*}{ Chile } & $\mathbf{P 1 0}$ & 0,2701 & - & 0,3019 & 0,2789 & 0,3264 & 0,2938 \\
\cline { 2 - 9 } & $\mathbf{Q 1}$ & 0,4392 & - & 0,4820 & 0,4476 & 0,4801 & 0,4476 \\
\cline { 2 - 9 } & $\mathbf{Q 4}$ & 0,5600 & - & 0,5778 & 0,5460 & 0,5769 & 0,5229 \\
\cline { 2 - 8 } & $\mathbf{P 9 0}$ & 0,6034 & - & 0,6223 & 0,6009 & 0,6312 & 0,5766 \\
\hline
\end{tabular}

\begin{tabular}{|c|c|c|c|c|c|c|c|}
\hline \multirow{4}{*}{ Colombia } & P10 & - & - & 0,2891 & 0,2615 & 0,3094 & 0,2594 \\
\hline & Q1 & - & - & 0,4004 & 0,4243 & 0,4450 & 0,4350 \\
\hline & Q4 & - & - & 0,4633 & 0,4760 & 0,4509 & 0,5143 \\
\hline & P90 & - & - & 0,5032 & 0,5005 & 0,4901 & 0,5941 \\
\hline \multirow{4}{*}{ Costa Rica } & P10 & - & - & - & 0,2406 & 0,2595 & 0,2352 \\
\hline & Q1 & - & - & - & 0,3883 & 0,4269 & 0,4083 \\
\hline & Q4 & - & - & - & 0,5015 & 0,4827 & 0,4915 \\
\hline & P90 & - & - & - & 0,5597 & 0,5499 & 0,5341 \\
\hline \multirow{4}{*}{ Rep. Dominicana } & P10 & - & - & - & - & - & 0,2173 \\
\hline & Q1 & - & - & - & - & - & 0,3973 \\
\hline & Q4 & - & - & - & - & - & 0,4355 \\
\hline & P90 & - & - & - & - & - & 0,5024 \\
\hline \multirow{4}{*}{ México } & P10 & 0,2736 & 0,2998 & 0,3611 & 0,3457 & 0,3587 & 0,3314 \\
\hline & Q1 & 0,4599 & 0,4752 & 0,5303 & 0,4861 & 0,5056 & 0,4891 \\
\hline & Q4 & 0,5487 & 0,4673 & 0,4844 & 0,4815 & 0,4871 & 0,4581 \\
\hline & P90 & 0,5728 & 0,5312 & 0,5428 & 0,5381 & 0,5346 & 0,5239 \\
\hline
\end{tabular}




\begin{tabular}{|l|c|c|c|c|c|c|c|}
\hline \multirow{5}{*}{ Panamá } & P10 & - & - & - & 0,2563 & - & - \\
\cline { 2 - 9 } & Q1 & - & - & - & 0,4341 & - & - \\
\cline { 2 - 9 } & Q4 & - & - & - & 0,5840 & - & - \\
\cline { 2 - 9 } & P90 & - & - & - & 0,5322 & - & - \\
\hline \multirow{5}{*}{ Perú } & P10 & 0,2590 & - & - & 0,3201 & 0,3394 & 0,3650 \\
\cline { 2 - 9 } & Q1 & 0,4310 & - & - & 0,5163 & 0,5205 & 0,5672 \\
\cline { 2 - 9 } & $\mathbf{Q 4}$ & 0,4844 & - & - & 0,4932 & 0,4785 & 0,5006 \\
\cline { 2 - 9 } & $\mathbf{P 9 0}$ & 0,5310 & - & - & 0,5640 & 0,5261 & 0,5472 \\
\cline { 2 - 9 } & $\mathbf{P 1 0}$ & - & 0,2549 & 0,2539 & 0,2324 & 0,2048 & 0,1959 \\
\cline { 2 - 9 } & $\mathbf{Q 1}$ & - & 0,4055 & 0,4161 & 0,3876 & 0,3854 & 0,3754 \\
\cline { 2 - 9 } & $\mathbf{Q 4}$ & - & 0,4589 & 0,4939 & 0,4101 & 0,5077 & 0,4712 \\
\cline { 2 - 8 } & $\mathbf{P 9 0}$ & - & 0,4800 & 0,5581 & 0,4319 & 0,5944 & 0,5445 \\
\hline
\end{tabular}

Fuente. Elaboración propia a partir de PISA 2000, 2003, 2006, 2009, 2012 y 2015

\section{CONCLUSIONES}

Los países de América Latina se encuentran entre los más inequitativos del mundo y sus sistemas educativos, con una alta segregación escolar, contribuyen a mantener y potenciar esas inequidades.

Este estudio ha analizado la evolución de la segregación escolar por nivel socioeconómico en varios países de la Región, específicamente en diez países cuyos datos están disponibles por haber participado en las diferentes ediciones de PISA. Las evidencias encontradas permiten ser moderadamente optimistas: en seis de los ocho países con suficientes datos la segregación parece tener una tendencia a la baja, especialmente en Argentina y Brasil; pero sube Colombia y Perú. Esta disminución es muy discreta en comparación con la alta segregación que presentan. Además, en la mayoría de los países en que la segregación disminuye, lo hace en los estudiantes de familias de mayor nivel socioeconómico, incrementándose en los de menor. Aunque faltan estudios al respecto, no sería aventurado hipotetizar que los sistemas educativos de América Latina se encuentran entre los más segregados del mundo.

Este hecho es altamente preocupante debido a las nefastas consecuencias que la segregación representa para la población escolar y el conjunto de la sociedad. Por una parte, porque afecta el desarrollo personal y social de los alumnos, en especial de aquellos más vulnerables o de sectores desfavorecidos. Pero no solo en esos casos, como muestra este estudio, también repercute en los estudiantes que proceden de familias de mayores recursos, por cuanto se les priva de la oportunidad de formarse en espacios más inclusivos y diversos, en términos de la composición sociocultural de los grupos donde se educan e interactúan. Por otra parte, la segregación escolar restringe el impacto del llamado efecto par, crecientemente reconocido en la comunidad educativa internacional por los beneficios que el aprendizaje y la enseñanza entre iguales genera, principalmente en 
contextos heterogéneos. Todo ello, redunda finalmente en experiencias de socialización y aprendizaje que limitan el potencial desarrollo de todos, y no solo de algunos, los con menor capital sociocultural como se tiende a argumentar. A este respecto, la investigación sobre la magnitud y evolución de la segregación escolar en América Latina deja abierto el camino para indagar en los efectos que este fenómeno tiene en los grupos de mayor nivel socioeconómico y cultural, un tema no estudiado en la región.

A través de este estudio vuelve a quedar de manifiesto la relación causal que existe entre los niveles de segregación escolar que exhiben los países y los modelos educativos de mercado con deficientes sistemas de regulación estatal, que han ido ganando terreno en la región a través de mecanismos y políticas que tienden a perpetuarlos en el tiempo, calando hondo en la cultura de la comunidad. Por poner algunos ejemplos, los mecanismos de selección al ingreso, el financiamiento por matrícula, el uso extendido de pruebas estandarizadas de resultados de aprendizaje, la publicación de rankings e incentivos asociados a resultados de las escuelas, que junto con fomentar la competitividad conducen a discriminar a los estudiantes con mayores dificultades sociales y académicas.

No cabe duda, que la segregación escolar, entendida como la distribución desigual de los estudiantes en las escuelas, en este caso por condiciones socioeconómicas, es una de las expresiones más elocuentes de la inequidad que caracteriza a los sistemas educativos de la región, situación que difícilmente se podrá revertir si no es abordada mediante políticas intersectoriales que ataquen sus múltiples causas. Una de ellas, como ya se ha señalado, tiene que ver con la segregación geográfica o residencial que afecta a amplios sectores de población, por lo cual la segregación escolar no puede ser vista de manera aislada como un fenómeno que atañe solo a educación, si se quiere tener éxito es necesario actuar en múltiples frentes.

No obstante lo anterior, tampoco se puede soslayar la responsabilidad que les cabe a los sistemas educativos en la producción de la segregación escolar y sus consecuencias. Por ello, un imperativo ético del estado y sus políticas educativas debe ser asumirla como problema y destinar los esfuerzos que sean necesarios para reducirla. Ello supone implementar políticas de equidad dirigidas a igualar la calidad de la educación que ofrecen las escuelas en que se concentran los estudiantes de sectores más desfavorecidos, que son quienes más necesitan una buena educación y los que reciben una de peor calidad.

En definitiva, es prioritario que los sistemas educativos de la Región desarrollen políticas públicas integrales de inclusión que reconociendo el valor de la diversidad para el aprendizaje y la convivencia social, promuevan la mixtura de estudiantes de diferentes condiciones socioeconómicas, culturales y capacidades en las escuelas.

\section{REFERENCIAS BIBLIOGRÁFICAS}

Agirdag, O., Van Avermaet, P. y Van Houtte, M. (2013). School segregation and math achievement: A mixed-method study on the role of self-fulfilling prophecies. Teachers College Record, 115(3), $1-50$.

Allen, R. y Vignoles, A. (2006). What should and index of school segregation measure? Londres: London School of Economics.

Arcidiácono, M., Cruces, G., Gasparini, L., Jaume, D., Serio, M. y Vázquez, E. (2014). La segregación escolar público-privada en América Latina. Santiago de Chile: Cepal. 
Ayscue, J. B. y Orfield, G. (2016). Perpetuating separate and unequal worlds of educational opportunity through district lines: school segregation by race and poverty. En P Noguera, J. Pierce y R. Ahram (Eds.), Race, Equity, and Education (pp. 45-74). Londres: Springer.

Backhoff, E. (2011). La inequidad educativa en México: Diferencias en el aprendizaje de la comprensión lectora en educación básica. Profesorado. Revista de Currículum y Formación de Profesorado, 15(3), 87-102.

Balarín, M. (2016). La privatización por defecto y el surgimiento de las escuelas privadas de bajo costo en el Perú. ¿Cuáles son sus consecuencias? RASE: Revista de la Asociación de Sociología de la Educación, 9(2), 181-196.

Bartholo, T. L. (2013). Measuring between-school segregation in an open enrollment system: the case of Rio de Janeiro. Journal of School Choice, 7(3), 353-371. https://doi.org/10.1080/15582 159.2013.808937

Bellei, C. (2013). El estudio de la segregación socioeconómica y académica de la educación chilena. Estudios pedagógicos (Valdivia), 39(1), 325-345.

Benavides, M., León, J. y Etesse, M. (2014). Desigualdades educativas y segregación en el sistema educativo peruano. Una mirada comparativa de las pruebas PISA 2000 y 2009. Lima: GRADE.

Benito, R. y Gonzàlez, I. (2007). Processos de segregació escolar a Catalunya. Barcelona: Mediterrània.

Böhlmark, A., Holmlund, H. y Lindahl, M. (2016). Parental choice, neighbourhood segregation or cream skimming? An analysis of school segregation after a generalized choice reform. Journal of Population Economics, 29(4), 1155-1190. https://doi.org/10.1007/s00148-016-0595-y

Burgess, S., Wilson, D. y Lupton, R. (2005). Parallel lives? Ethnic segregation in schools and neighbourhoods. Urban Studies, 42(7), 1027-1056.

Duarte, J., Bos, M. S. y Moreno, J. M. (2012). Calidad, igualdad y equidad en la educación colombiana. Washington, DC: Bando Interamericano de Desarrollo.

Duncan, O. y Duncan, B. (1955). A methodological analysis of segregation indexes. American Sociological Review, 20, 210-217. https://doi.org/10.2307/2088328

Dupriez, V. (2010). Methods of grouping learners at school. París: UNESCO.

Echenique, F. y Fryer J. (2007). A measure of segregation based on social interactions. The Quarterly Journal of Economics, CXXII (2), 441-485. https://doi.org/10.1162/qjec.122.2.441

Elacqua, G. (2012). The impact of school choice and public policy on segregation: Evidence from Chile. International Journal of Educational Development, 32(3), 444-453.

Entorf, H. y Lauk, M. (2008). Peer effects, social multipliers and migrants at school: An international comparison. Journal of Ethnic and Migration Studies, 34(4), 633-654.

Frankel, D. y Volij, O. (2011). Measuring school segregation. Journal of Eonomic Theory, 146(1), 1-38. https://doi.org/10.1016/j.jet.2010.10.008

Gasparini, L., Jaume, D., Serio, M. y Vázquez, E. (2011). La segregación entre escuelas públicas y privadas en Argentina. Reconstruyendo la evidencia. Desarrollo Económico, 51(202), 189-219

Goldstein, H. y Noden, P. (2003). Modelling social segregation. Oxford Review of Education, 29(2), 225-237. https://doi.org/10.1080/0305498032000080693

Gorard, S. (2006). What does an index of school segregation measure? A commentary on Allen and Vignoles. York: Department of Educational Studies.

(1998). Social movement in undeveloped markets: An apparent contradiction. Educational Review, 50(3), 249-258. https://doi.org/10.1080/0013191980500304

Gorard, S. y Fitz, J. (2000). Investigating the determinants of segregation. Research Papers in Education, 15(2), 115-132. https://doi.org/10.1080/026715200402452

Gorard, S. y Smith, E. (2004). An international comparison of equity in education systems. Comparative Education, 40(1), 15-28. https://doi.org/10.1080/0305006042000184863

Gorard, S. y Taylor, C. (2002). What is segregation? A comparison of measures in terms of 'strong' and 'weak' compositional invariance. Sociology, 36(4), 875-895. https://doi. 
org/10.1177/003803850203600405

Gramberg, P. (1998). School segregation: The case of Amsterdam. Urban Studies, 35(3), 547-564. https://doi.org/10.1080/0042098984907

Harker, R. y Tymms, P. (2004). The effects of student composition on school outcomes. School effectiveness and school improvement, 15(2), 177-199.

Harris, R. (2012). Local indices of segregation with application to social segregation between London's secondary schools, 2003-08/09. Environment and Planning A, 44(3), 669-687. https:// doi.org/10.1068/a44317

Hutchens, R. (2004). One measure of segregation. International Economic Review, 45(2), 555-578. . https://doi.org/10.1111/j.1468-2354.2004.00136.x

(2001). Numerical measures of segregation: desirable properties and their implications. Mathematical Social Sciences, 42(1), 13-29. https://doi.org/10.1016/s0165-4896(00)00070-6

Johnston, R., Wilson, D. y Burgess, S. (2004). School segregation in multiethnic England. Ethnicities, 4(2), 237-265. https://doi.org/10.1177/1468796804042605

Kao, G. y Thompson, J. S. (2003). Racial and ethnic stratification in educational achievement and attainment. Annual Review of Sociology, 29, 417-442.

Karsten, S. (2010). School segregation. En OECD, Equal opportunities?: The labour market integration on the children of immigrants (pp. 193-209). París: OECD Publishing. https://doi.org/10.1787/9789264086395-en

Karsten, S., Felix, C., Ledoux, G., Meijnen, G., Roeleveld, J. y Schooten, E. (2006). Choosing segregation or integration? The extent and effects of ethnic segregation. Education and Urban Society, 38(2), 228-247. https://doi.org/10.1177/0013124505282606

Krüger, N. (2014). Más allá del acceso: segregación social e inequidad en el sistema educativo argentino. Cuadernos de Economía, 33(63), 513-542. https://doi.org/10.15446/cuad. econ.v33n63.45344

Lieberson, S. (1981). An asymmetrical approach to segregation. En C. Peach (Ed.), Ethnic segregation in cities (pp. 61-83). Londres: Croom-Helm.

Massey, D. S. y Denton, N. A. (1988). The dimensions of residential segregation. Social Forces, 67(2), 281-315. https://doi.org/10.2307/2579183

McArdle, N. y Acevedo-Garcia, D. (2017). Consequences of Segregation for Children's Opportunity and Wellbeing. Cambridge, MA: Harvard University.

Morgan, B. S. (1983). A distance-decay based interaction index to measure residential segregation. Area, 15, 211-217.

Murillo, F. J. (2016). Midiendo la segregación escolar en América Latina. Un análisis metodológico utilizando el TERCE. REICE. Revista Iberoamericana sobre Calidad, Eficacia y Cambio en Educación, 14(4), 33-60. https://doi.org/10.15366/ reice2016.14.4.002

Murillo, F.J. y Martínez-Garrido, C. (2018). Magnitud de la segregación escolar por nivel socioeconómico en España y sus Comunidades Autónomas y comparación con los países de la Unión Europea. RASE: Revista de la Asociación de Sociología de la Educación, en prensa. . (2017). Segregación escolar por nivel socioeconómico en Ecuador y sus provincias. RUNAE. Revista Científica de Investigación Educativa de la UAE, 1(2),

(2017). Estimación de la magnitud de la segregación escolar en América Latina. Magis, Revista Internacional de Investigación en Educación, 9(19), 11-30. https://doi. org/10.11144/Javeriana.m9-19.emse

Murillo, F. J., Martinez-Garrido, C. y Belavi, G. (2017). Segregación escolar por origen nacional en España. OBETS. Revista de Ciencias Sociales, 12(2), 395-432. https://doi.org/10.14198/ OBETS2017.12.2.04

Nechyba, T. (2006). Income and peer quality sorting in public and private schools. Handbook of the Economics of Education, 2, 1327-1368. https://doi.org/10.1016/ 


\section{S1574-0692(06)02022-8}

Noden, P. (2000). Rediscovering the impact of marketisation: Dimensions of social segregation in England's secondary schools, 1994-99. British Journal of Sociology of Education, 21(3), 371-390. https://doi.org/10.1080/713655353

Oberti, M. (2012). Chicago et Paris, Métropoles de la ségrégation scolaire? París: La vie des idées

OCDE. (2016). Programme of international student assessment (PISA 2015). París: OCDE.

Orfield, G. (2001). Schools more separate: Consequences of a decade of resegregation. Cambridge, MA: Harvard University.

Owens, A., Reardon, S.F. y Jencks, C. (2016). Income Segregation between Schools and School Districts. Los Angeles, CA: Stanford Center for Education Policy Analysis.

Reardon, S. F. y Owens, A. (2014). 60 Years after Brown: Trends and consequences of school segregation. Annual Review of Sociology, 40, 199-218.

Rumberger, R. W., \& Palardy, G. J. (2005). Does segregation still matter? The impact of student composition on academic achievement in high school. Teachers college record, 107(9), 1999.

Saporito, S. (2003). Private choices, public consequences: Magnet school choice and segregation by race and poverty. Social Problems, 50(2), 181-203.

Siddiqui, N. (2017). Socio-economic segregation of disadvantaged children between schools in Pakistan: comparing the state and private sector. Educational Studies, 43(1), 1-19. https://doi.org/10.1080/03055698.2016.1277139

Stroub, K. J. y Richards, M. P. (2013). From resegregation to reintegration trends in the racial/ethnic segregation of metropolitan public schools, 1993-2009. American Educational Research Journal, 50(3), 497-531.

Taylor, C. y Gorard, S. (2001). The role of residence in school segregation: placing the impact of parental choice in perspective. Environment and Planning A, 33(10), 1829-1852.

Valenzuela, J. P. (2008). Segregación en el Sistema Escolar Chileno: en la búsqueda de una educación de calidad en un contexto de extrema desigualdad. Santiago de Chile: Universidad de Chile.

Valenzuela, J. P., Bellei, C. y De los Ríos, D. (2014). Socioeconomic school segregation in a market-oriented educational system. The case of Chile. Journal of Education Policy, 29(2), 217-241.

(2008). Evolución de la segregación socioeconómica de los estudiantes chilenos y su relación con el financiamiento compartido. Santiago de Chile: Fonide, Ministerio de Educación de Chile.

(2010) Segregación Escolar en Chile. En S. Martinic y G. Elacqua (Eds.), ¿Fin de ciclo? Cambios en la gobernanza del sistema educativo. OREALC/UNESCO - P. Universidad Católica de Chile.

Van Ewijk, R. y Sleegers, P. (2010). Peer ethnicity and achievement: A meta-analysis into the compositional effect. School Effectiveness and School Improvement, 21(3), 237-265. (2010). The effect of peer socioeconomic status on student achievement: A metaanalysis. Educational Research Review, 5(2), 134-150.

Vázquez, E. (2012). Segregación escolar por nivel socioeconómico. Midiendo el fenómeno y explorando sus determinantes. Buenos Aires: CEDLAS.

Willms, J. D. (1986). Social class segregation and its relationship to pupils' examination results in Scotland. American Sociological Review, 51(2), 224-241. https://doi.org/10.2307/2095518

Zhou, Y., Cai, T. Y Wang, D. (2016). Social Segregation in Hong Kong's Schools: 2000-2012. Chinese Sociological Review, 48(3), 248-270. https://doi.org/10.1080/21620555.2016.1166340

Zimmerman, D.J. (2003). Peer effects in academic outcomes: Evidence from a natural experiment. The Review of Economics and Statistics, 85, 9-23. 
